\title{
Diseño e implementación de un control mecánico con cables tipo Push-Pull para un banco de pruebas en tierra de motores PT6*
}

| Fecha de recibido: 8 de febrero del 2020 | Fecha de aprobación: 29 de abril del 2020 |

\author{
Juan Sebastián Solís Chaves \\ Ph. D. en Ingeniería de la Energía \\ Docente Escuela Colombiana de Carreras \\ Industriales (ECCI). Grupo de investigación: GIATME \\ Rol de investigador: intelectual, \\ experimental, comunicativo \\ https://orcid.org/0000-0002-3211-3398 \\ $\triangle$ jsolisc@ecci.edu.co
}

\section{Jeison Ferney Barrios Rojas \\ Estudiante de pregrado en Ingeniería Mecánica \\ Escuela Colombiana de Carreras Industriales (ECCI) \\ Rol de investigador: experimental, comunicativo https://orcid.org/0000-0003-1457-5246 $\bowtie$ jeisonf.barriosr@ecci.edu.co}

\section{Nelson Arturo Jiménez Acuña}

Técnico en Mantenimiento Aeronáutico

Fuerza Aérea Colombiana

Rol de investigador: intelectual, experimental https://orcid.org/0000-0003-4076-4809 $\triangle$ naja52@yahoo.com

\section{César Geovany Quiroga Vargas}

Estudiante de pregrado en Ingeniería Mecánica Escuela Colombiana de Carreras Industriales (ECCI)

Rol de investigador: experimental, comunicativo https://orcid.org/0000-0002-1265-8825 $\triangle$ cesarg.quirogav@ecci.edu.co

\section{Ángela Paola Sánchez Alba}

Estudiante de pregrado en Ingeniería Mecánica

Escuela Colombiana de Carreras Industriales (ECCI) Rol de investigador: experimental https://orcid.org/0000-0002-5223-6678 $\bowtie$ angelap.sancheza@ecci.edu.co

* Artículo de investigación derivado del proyecto Uso de Biocombustibles en Aeronaves de la Fuerza Aérea Colombiana. Financiado por Colciencias. Código 56752

Cómo citar este artículo: Solís Chaves, J. S., Barrios Rojas, J. F., Jiménez Acuña, N. A., Quiroga Vargas, C. G., \&. Sánchez Alba, A. P. (2020). Diseño e implementación de un control mecánico con cables tipo Push-Pull para un banco de pruebas en tierra de motores PT6. Revista Ciencia y Poder Aéreo, 15(1), 135-151. https://doi.org/10.18667/cienciaypoderaereo.646 


\section{Diseño e implementación de un control mecánico con cables tipo Push-Pull para un banco de pruebas en tierra de motores PT6}

Resumen: Este artículo presenta el diseño y la implementación de un control mecánico por medio de cables y palancas, también conocidos como cables tipo Push-Pull para los comandos de vuelo de un motor PT6 61A. El propósito es controlar la condición del combustible, potencia del motor y posición angular de la hélice con el motor operando en tierra, mientras es controlado desde un banco de pruebas ubicado en el Comando Aéreo de Mantenimiento (CAMAN). La implementación del controlador se realizó con materiales aeronáuticos reciclados que, no obstante, proporcionan un margen de seguridad al operador y, así, salvaguardan la integridad del motor. Para llevar a cabo la práctica de este diseño se aplicó una metodología empírico-analítica en la que destacan, principalmente, la caracterización del motor, adquisición, adecuación, construcción de los elementos de fijación, instalación y ajuste de las palancas mecánicas de cada comando. Con el objetivo de probar el desempeño del controlador mecánico propuesto con cables tipo Push-Pull, se realizaron pruebas con mezclas de combustible tradicional (JET-A1) y biodiesel. Por último, los datos de temperatura, velocidad, torque y porcentaje (NG) adquiridos en cada prueba facilitaron la comparación del funcionamiento del motor y el correcto desempeño del controlador.

Palabras clave: Biocombustibles; cable de control mecánico; comandos de vuelo; palancas de control; PT6; turbinas aeronáuticas.

\begin{abstract}
This paper presents the design and deployment of a mechanical control with cables and levers, also known as push-pull cables, for flight commands in a PT6 61A engine. The purpose of the study is to control the variables fuel condition, engine power, and propeller angular position while having the engine operating on the ground and being controlled from a test bench located at the Air Maintenance Command (CAMAN, in Spanish). The implementation of the controller was carried out with recycled aeronautical materials that, nevertheless, provide a margin of safety for the operator and ensure the integrity of the engine. An empirical-and-analytical methodology was applied to carry out this design, in which the characterization of the engine, the acquisition, adaptation and construction of the fixing elements, as well as the installation, and the adjustment of the mechanical levers of each command became key aspects. In order to test the performance of the proposed mechanical control, tests were carried out with mixtures of traditional fuel (JET-A1) and biodiesel. The data on temperature, speed, torque and percentage (NG) in each test facilitated the comparison of the operation of the engine and the correct performance of the controller.
\end{abstract}

Keywords: Biofuels; Mechanical Control Cable; Flight Commands; Control Levers; PT6; Aeronautical Turbines.

Resumo: Este artigo apresenta o desenho e a implementação de um controle mecânico por meio de cabos e alavancas, também conhecidos como cabos Push-Pull, para comandos de vôo de um motor PT6 61A. O objetivo é controlar a condição do combustível, a potência do motor e a posição angular da hélice com o motor operando em terra, enquanto é controlado a partir de um banco de testes localizado no Comando Aéreo de Manutenção (CAMAN). A implementação do controlador foi realizada com materiais aeronáuticos reciclados, que, no entanto, fornecem uma margem de segurança para o operador e, desta forma, salvaguardar a integridade do motor. Para realizar a prática do desenho, foi adotada uma metodologia empírico-analítica, na qual se destacam a caracterização do motor, aquisição, adaptação, construção dos elementos de fixação, instalação e ajuste das alavancas mecânicas de cada comando. Com o objetivo de testar o desempenho do controlador mecânico proposto com cabos Push-Pull, foram realizados testes com misturas de combustível tradicional (JET-A1) e biodiesel. Finalmente, os dados de temperatura, velocidade, torque e porcentagem (NG) adquiridos em cada teste, facilitaram a comparação da operação do motor e o correto desempenho do controlador.

Palavras-chave: Biocombustíveis; Cabo de controle mecânico; Comandos de vôo; Alavancas de controle; PT6; Turbina aeronáutica. 


\section{Introducción}

Actualmente, la Fuerza Aérea Colombiana (FAC) adelanta un proyecto de investigación para el aprovechamiento y la utilización de biocombustibles en varios tipos de motores aeronáuticos de su flota de aeronaves, busca cambiar el uso de los combustibles tradicionales derivados del petróleo a biocombustibles, con el fin de reducir total o parcialmente los gases contaminantes emitidos a la atmósfera. Con tal propósito, este artículo describe la implementación del control mecánico del motor PT6, el cual será operado en tierra desde un banco de pruebas que se encuentra instalado en el Comando Aéreo de Mantenimiento (CAMAN) de la FAC en Madrid, Cundinamarca. La adecuación se llevará a cabo a través de cables de control tipo Push-Pull y palancas mecánicas dispuestas para cada uno de los comandos de vuelo, que son potencia del motor, condición del combustible y posición angular de la hélice. Estos controles se adecuaron en gran parte usando materiales reutilizados, los mandos han sido conectados mediante cables de control mecánico, gracias a que presentan una alta precisión y confiabilidad, en contraste con su bajo costo cuando son comparados con otras alternativas típicas en aplicaciones aeronáuticas (Guayacol, 2013; Cables y Controles, 2020).

El control mecánico de los comandos básicos de vuelo propuesto, recrea las operaciones reales de la aeronave de acuerdo con los múltiples regímenes establecidos por la casa fabricante, Pratt \& Whitney Canada (Pratt \& Whitney Canada, 2007; 2019), con lo que se obtiene un control total del motor, lo cual permite que las mediciones análogas determinen la eficiencia energética de las diferentes mezclas entre biodiesel y combustible JET A-1 con la precisión necesaria para realizar comparaciones altamente confiables que puedan ser replicadas cuantas veces sea necesario para los distintos propósitos de análisis estadístico y de desempeño del motor. Este proyecto es realizado por CAMAn, la Escuela de Suboficiales de la Fuerza Aérea (ESUFA) y la universidad ECCI, quienes dentro del equipo de trabajo cuentan con personal de soporte para enfrentar las posibles emergencias durante la operación del motor.

A su vez, la investigación busca comprobar el desempeño del modelo PT6 61A y determinar la cantidad de emisiones de Gases de Efecto Invernadero (GEI), al realizar pruebas con diferentes mezclas de biodiesel (BioD, 2015) y JET A-1 en distintas proporciones. Por ejemplo, un $95 \%$ de JET A-1 y un $5 \%$ de BioD, o con un $85 \%$ de JET A-1 y un $15 \%$ de BioD, o bien, con una proporción de $75 \%$ JET A-1 y $25 \%$ BioD (Sánchez, 2020). Además, se han tenido en cuenta las propiedades físico-químicas en los combustibles empleados en las pruebas (Mayorga Betancourt et al., 2019), así como la eficiencia, concentración óptima y posibles desventajas e impactos ambientales. Por tales motivos y para mayor claridad del lector, se presenta un corte transversal del motor a ser controlado, como se muestra en la figura 1.
Figura 1. Esquema del motor PT6 Fuente: Bayona-Roa et al. (2019).

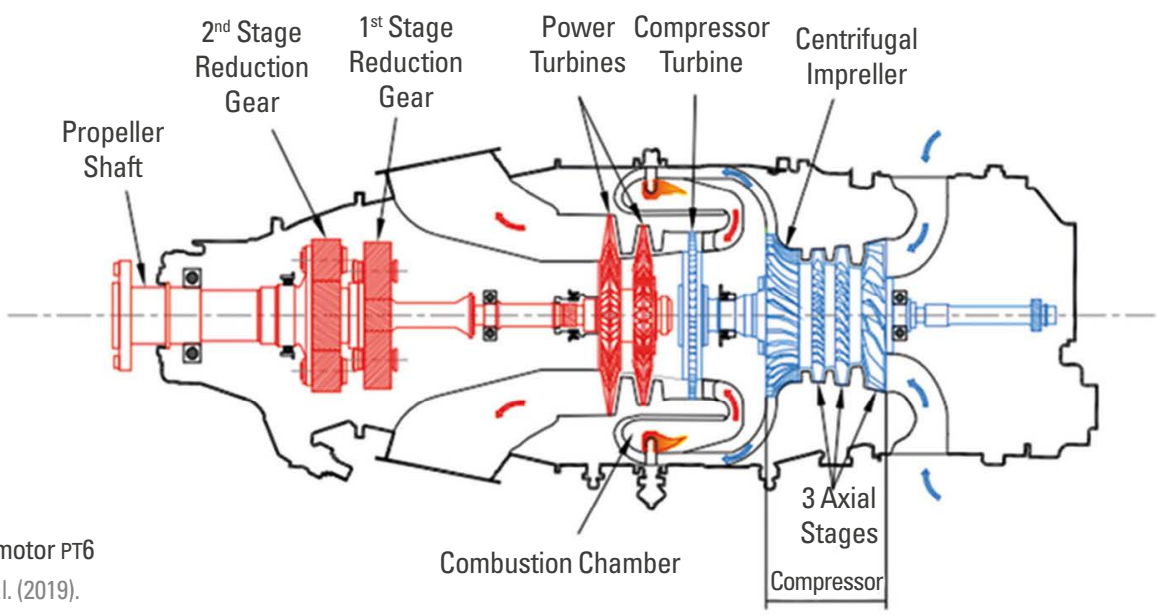

Revista Ciencia y Poder Aéreo | vol. 15 n. 1 | enero-junio del 2020 | pp. 135-151 | ISSN 1909-7050 E-ISSN 2389-9468 | https://doi.org/10.18667/cienciaypoderaereo.646 
El anterior es un motor turbo-hélice modular con tipo de turbina libre, fabricado por Pratt \& Whitney, modelo PT6 61A, tamaño medio. Dentro de la aviación, su aplicabilidad se presenta en los aviones Piper Cheyenne, como el PA-42-720 (FAC, s.f.), en el que la potencia del eje de su motor es de 850 SHP ${ }^{1}$ certificados según el documento A23SO. Lo dicho representa una potencia aplicable a una aeronave de $720 \mathrm{SHP}$, en la cual la turbina pesa aproximadamente $220 \mathrm{~kg}$, su configuración para avión, incluyendo la hélice, es simple y el peso puede llegar a ser de $300 \mathrm{~kg}$. Posee una longitud de $72,09 \mathrm{~cm}$, un diámetro nominal de $18,29 \mathrm{~cm}$ y un radio máximo, excluyendo los ductos de escape, de 12,84 cm (Pratt \& Whitney Canada, 2007; 2019).

El artículo presenta un resumen de estudios previos enfocados en el comando del motor PT6, luego se describe el diseño del controlador mecánico por medio de cables de control tipo Push-Pull, en seguida se hace el listado y la descripción de los componentes necesarios para la implementación del controlador, explicando la adquisición, adecuación y construcción de los elementos de fijación. Acto seguido, se describe la instalación y el ajuste de las palancas mecánicas de cada uno de los comandos, después se presentan los resultados experimentales, que muestran el movimiento de la palanca, los cables de cada comando, las tablas de reglajes y sus porcentajes de error cuando se realiza una corrida experimental del motor con combustible tradicional y con la mezcla de biocombustible. Por último, se muestran las observaciones y recomendaciones a tener en cuenta, así como las conclusiones de este proyecto.

\section{Estudios previos}

Los esfuerzos se enfocaron en encontrar trabajos que dieran cuenta del diseño y control de los comandos de vuelo en bancos de pruebas, la inclusión de los biocombustibles en el sector aeronáutico en Colombia y

1 Shaft Horse Power. en artículos que simularán el desempeño del motor PT6 al ser operado con biocombustibles. En primera instancia, se encontró un documento titulado Diseño de un banco de pruebas para motores PT6 T-3, realizado por la Universidad San Buenaventura. Para la fecha de presentación de esa tesis, no se contaba con la facilidad de acceso a bancadas experimentales para probar motores PT6, por tanto, los autores decidieron diseñar un banco de pruebas con el fin de realizar overhaul y pruebas post-overhaul con el motor emplazado en tierra, y así mismo disminuir los costos asociados a la reparación y mantenimiento del motor (Ferreira et al., 2008).

El diseño de banco de pruebas en el documento nombrado, será utilizado para distintas series de motores PT6, y también presta servicio para los motores tipo PWC PT6 de Pratt \& Whitney Canada, que se encuentran en Colombia para realizar las actividades de mantenimiento y verificación necesarias (Ferreira et al., 2008).

Asimismo, se hizo una búsqueda bibliográfica referente a los controles de vuelo del motor PT6 que serán implementados en la cabina del banco de pruebas, indagación que señaló los métodos de conexión de las palancas para efectuar el manejo del motor y su función de control. La palanca de estado de combustible tiene como función el avance y parada de combustible, también funciona como un tope inactivo para la barra de interconexión de la unidad de control de combustible $\mathrm{FCU}^{2}$ (Pratt \& Whitney Canada, s.f.).

Ahora bien, la palanca de control de potencia controla la potencia del motor en todo el rango, desde el máximo de despegue hasta el retroceso completo. De igual forma, elige el paso de la hélice (control Beta) desde la selección inversa hasta la velocidad constante de RPM seleccionada por la palanca de control de la hélice de la cabina (velocidad de la hélice) (Pratt \& Whitney Canada, s.f.).

Por otra parte, al investigar sobre el panorama actual de la aeronáutica en Colombia, se encontró que la Corporación de la Industria Aeronáutica Colombiana

2 Fuel Control Unit. 
(CIAC) ha sido una entidad protagonista en el rápido desarrollo del sector aeronáutico en el país durante los últimos años, ya que se encarga de realizar proyectos de fabricación y modernización de aeronaves. Los autores de este documento piensan que un vínculo estrecho con la CIAC, dado lo propuesto en este proyecto y lo que el controlador aporta específicamente, generará un aporte significativo hacia la disminución de los índices de contaminación por emisión de Gases de Efecto Invernadero (GEI) en el sector aeronáutico, al migrar a los combustibles ecológicos, ya sea en la flota de aviones de uso civil o en los de uso militar (Meisel, 2014; Semana, 2018; Delgado, 2018).

Por último, durante esta etapa de la investigación se localizó un artículo titulado Simulación computacional del motor de turbina de gas PT6A funcionando con diferentes mezclas de biodiesel: un análisis de respuesta transitoria, realizado por la universidad ECCI, en el cual se describe la solución aerotermodinámica que ocurre en las turbinas a gas, mediante un modelo matemático en el que se utiliza un procedimiento numérico. Este modelo fue realizado en Matlab-Simulink ${ }^{\circledR}$, empleando un enfoque en bloques para simular marchas transitorias del motor PT6A que funciona con mezclas de JET A-1 y biodiesel, obteniendo así las variables termodinámicas necesarias (Bayona-Roa et al., 2019).

\section{Metodología}

Para el proyecto propuesto se optó por usar una metodología empírico-analítica, que ha sido dividida en cuatro partes consideradas fundamentales para la realización del diseño de un control mecánico por medio de cables Push-Pull. La primera es la identificación de los comandos para controlar en el motor y su respectiva función, esto se realiza con el fin de determinar la medida para los cables de control mecánico y la ubicación del comando en el motor; la segunda etapa escoge la ruta de los cables de control mecánico desde la cabina de control hasta el PT6; la tercera fase comprende la elaboración de elementos de fijación para el cable de control mecánico en el motor y las palancas, para evitar así la flexión, la curvatura excesiva del cable o algún otro factor que disminuya el control del mismo. En el cuarto momento, se realiza la instalación de las palancas de control en la cabina del banco de pruebas desde el que se controla el motor PT6, se establecen los reglajes experimentales de cada uno de los comandos y se determina el porcentaje de error con respecto a los valores teóricos. Al final, se diseñaron dos experimentos comparativos que dan cuenta del desempeño seguro del controlador de control mecánico.

\section{Diseño del controlador para cables de control mecánico}

Para el diseño del controlador mediante cables de control mecánico desde banco de pruebas, se establece un sistema de comandos simples, pero con la precisión suficientemente puntual para reducir el rango de error que se pueda presentar en el desarrollo de las pruebas y en la toma de datos experimentales de las variables termodinámicas de interés. Se buscó implementar un sistema de palancas, las cuales fueran capaces de halar y empujar (Push-Pull) un cable de control mecánico, utilizados frecuentemente en aviación. Este tipo de cables presentan una serie de características que los hacen aptos para este tipo de operación, por ejemplo, su longitud y su calibre.

Gracias al recubrimiento de protección presente en el cable, fue posible asegurarlo a la bancada del motor y a la base del banco de pruebas, restringiendo el movimiento angular no deseado de este y evitando así la pérdida de movimiento lineal. Además, el cable tiene instaladas unas terminales tipo rótula que permiten el acople de los cables a los comandos en el motor y el movimiento angular existente en cada acople, maximizando de este modo su rango de acción con una precisión bastante fina. Las palancas de aluminio empleadas para esta labor poseen una serie de topes internos que se pueden manipular para ofrecer un movimiento angular acorde su desplazamiento lineal, así 
mismo cuentan con una lámina interna capaz de transmitir el movimiento total al cable de control mecánico, evitando la flexión interna en el mecanismo y obteniendo la precisión suficiente en el movimiento al momento de realizar los ensayos en tierra desde el banco de pruebas.

\section{Identificación de los comandos a controlar en el motor y su función}

Para iniciar la instalación del control de comandos del motor PT6 mediante los cables de control y las palancas de accionamiento mecánico, se debe identificar la ubicación de los comandos del motor PT6 y su objetivo de control como se describe a continuación:

\section{El control de vuelo en el motor PT6}

Los comandos mecánicos que deben ser trasladados de la cabina de vuelo al banco de pruebas son el mando de inyección de combustible, el mando de control de hélice (necesario para la prueba con carga mecánica) y el mando de potencia (mínima, máxima y reversible), los cuales son controlados mediante cables de control mecánico y sus respectivas palancas.

Estos cables de control mecánico están conectados normalmente entre los mandos que se encuentran en el pedestal de la cabina de vuelo, las diferentes secciones del motor (Generación de Gases -NG-, Generación de Potencia - NF-, Generación en la Hélice -NP-) y un conjunto de control de empuje o tracción, montado en la parte derecha longitudinal del motor (Pratt \& Whitney Canada, s.f.). Al ser los elementos encargados de realizar las acciones de control, deben contar con la precisión suficiente para brindar la confiabilidad requerida durante las pruebas con combustible JET A-1 y biocombustibles, como se presenta en la figura 2 .

La palanca que indica el estado de combustible en la cabina está conectada a través de los enlaces del fuselaje a una palanca combinada y a un mecanismo de parada en la parte superior de la unidad de control de combustible; el cual está conectado mediante el enlace de la FCU a la palanca de corte de combustible en el lateral de la unidad. Dicha palanca de avance y parada también funciona como un tope inactivo para la barra de interconexión de la FCU (Badger et al., 1994; Pratt \& Whitney Canada, 2006), tal como se observa en la figura 3.

A su vez, la palanca de control de potencia en la cabina está conectada con los enlaces del fuselaje al ensamblaje cambox, montado en frente de la FCU, controla la potencia del motor en todo el rango, desde el máximo de despegue hasta el retroceso completo. También elige el paso de la hélice (control Beta), desde la selección inversa hasta la velocidad constante de rpm seleccionada por la palanca de control de la hélice de la cabina (velocidad de la hélice) (Pratt \& Whitney Canada, 2006; Guimarães, 2015). Véase la figura 3.

Figura 2. Secciones del motor PT6

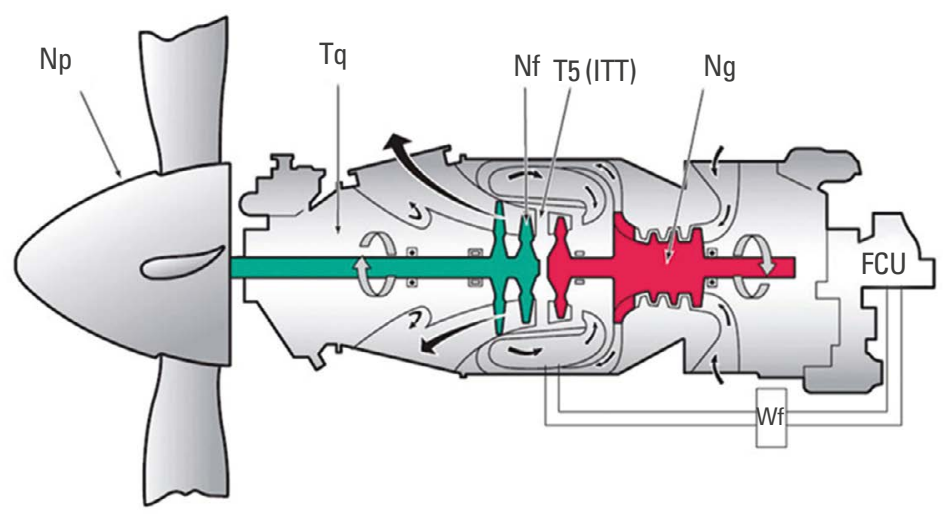

Fuente: Pratt \& Whitney Canada (s.f.). 


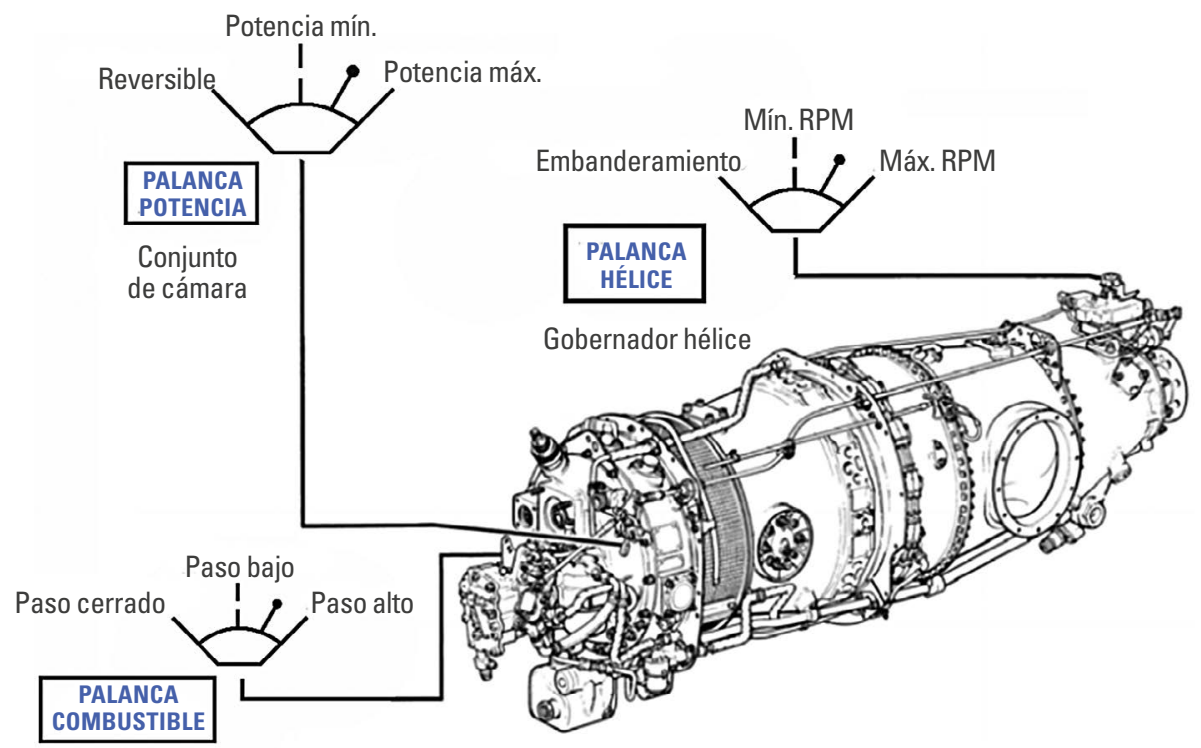

Figura 3. Mandos de control

Fuente: adaptada de Pratt \& Whitney Canada (s.f.).

El accionamiento de la FCU está conectado a través de una varilla de interconexión, ajustable a la palanca de control de esta unidad. En condiciones de funcionamiento normales, el movimiento de la palanca de control de potencia mueve el seguidor de leva a través de la ranura de la palanca de control de la hélice. Durante el funcionamiento normal de la hélice, desde el ralentí en tierra hasta el despegue, el pasador de palanca del seguidor de leva se mueve en la ranura circunferencial de la leva, pero no imparte movimiento a la palanca de control de la hélice (Pratt \& Whitney Canada, 2006).

\section{Controles de la cabina de vuelo}

En la actualidad, los motores PT6A utilizan el sistema de gestión de la potencia del motor y la condición del control de hélice, para obtener la capacidad de vuelo óptimo del avión, así como también la correspondiente capacidad de manejo en tierra. Las funciones de la central eléctrica normalmente se ordenan desde la cabina, por medio de tres palancas de control: palanca de potencia, palanca de apertura y cierre de combustible, y palanca de apertura y cierre de flujo de aceite para realizar el cambio de paso en la hélice, o también llamada palanca de hélice, y cables de control mecánico Push-Pull (Pratt \& Whitney Canada, s.f.; Méndez, 2011).

Las palancas están conectadas mediante cables de control mecánico a los mandos que se encuentran en el motor. La palanca de potencia tiene como función elevar la aceleración del motor, realizar el reversible, y a su vez, modificar el ángulo de ataque de la hélice; la palanca de combustible da el paso de este al motor, abriendo o cerrando su acceso; y la palanca de la hélice controla su velocidad y la rotación de las aspas (Pratt \& Whitney Canada, s.f.).

En algunos bancos de prueba de motores, en ocasiones para realizar el control en uno de ellos, solo basta con una palanca de control para manipular la condición de combustible, regular las rpm y manipular el control de potencia (Pratt \& Whitney Canada, s.f.; Guimarães, 2015). La siguiente tabla da cuenta de los comandos de control presentes en diferentes partes del motor, su ubicación y la función que cumple cada uno. 
Tabla 1

Ubicación y funciones de los comandos del control de vuelo motor PT6

\begin{tabular}{l|l|l}
\multicolumn{1}{c|}{ Control } & \multicolumn{1}{c|}{ Ubicación } & \multicolumn{1}{c}{ Función del control } \\
\hline Condición de combustible & Unidad de control de combustible (control de arranque). & Corte de combustible y velocidad de ralentí. \\
\hline Potencia & Unidad de control de combustible. & Velocidad del generador de gases. \\
\hline Hélice & Gobernador de la hélice. & Velocidad de hélice y rotación de las aspas. \\
\hline
\end{tabular}

Fuente: Pratt \& Whitney Canada (s.f.).

\section{Trazar la ruta para el cable de control mecánico}

En la siguiente figura se muestra el trazado final del cable de control mecánico desde el banco de prueba hasta el motor, con el fin de lograr una adecuada tensión en el cable de control sin que se generen curvaturas que afecten su funcionamiento:
En esta etapa se realiza la compra del cable de control mecánico, teniendo en cuenta las siguientes consideraciones: desde el banco de prueba hasta el motor se utiliza un (1) cable de control mecánico de 14 metros de longitud para el control del comando de la hélice, y dos (2) cables de 12 metros para el control de los comandos de combustible y de potencia, ambos con un calibre $3 / 8$ in $(9,53 \mathrm{~mm})$. Los cables se

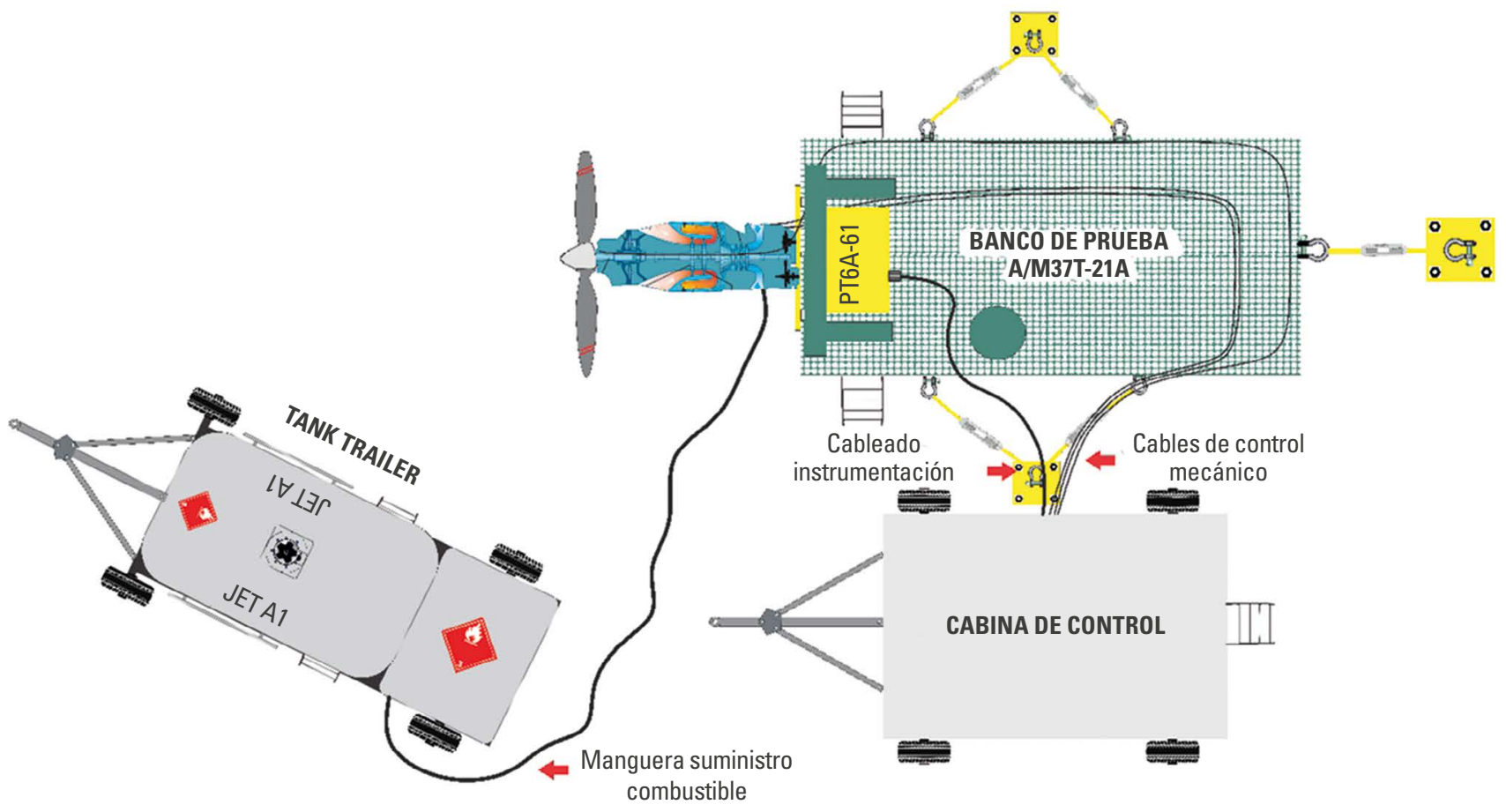

Figura 4. Trazado final del cable de control mecánico

Fuente: elaboración propia. 
posicionan en la plataforma donde están los soportes del motor y se aseguran con pasamuros para impedir su curvatura y posible desplazamiento. De igual forma, y para facilitar la sujeción tanto en los acoples del motor como en las terminales de las cajas de comandos, fue necesario implementar una terminal tipo rótula en las extremidades de los cables, estos deben ser capaces de proporcionar un agarre fijo, pero con un ángulo variable para evitar que se pueda interrumpir la transmisión del movimiento.

El cable de este tipo se elige según su disponibilidad comercial, tiempos de entrega, facilidad de montaje y ensamble. De manera adicional, se seleccionaron los cables debido a su recubrimiento, ya que tienen una camisa de trabajo de caucho que protege el interior de la guaya, con lo que se limita su exposición a elementos que puedan debilitarlos o, incluso, fragmentarlos. Otras características son su funcionamiento suave y continuo, resistencia y rigidez, pues a la vez admite curvaturas fuertes y cerradas (Cables y Controles, 2020).

Se muestra en la figura 5 la ruta de los cables de control mecánico desde la cabina hasta el motor, en primera instancia, se realiza la sujeción de los cables mediante bridas plásticas.

Dado que al momento de la instalación de las palancas no fue necesario cambiar la ruta de los cables, se procedió a realizar los elementos de fijación en el motor (pasamuros), para evitar la pérdida de señal mecánica en el cable al momento de ser accionado, y a la par, que el motor pueda funcionar sin que las vibraciones emitidas por este afecten el desempeño de los cables, véase la figura 6 .

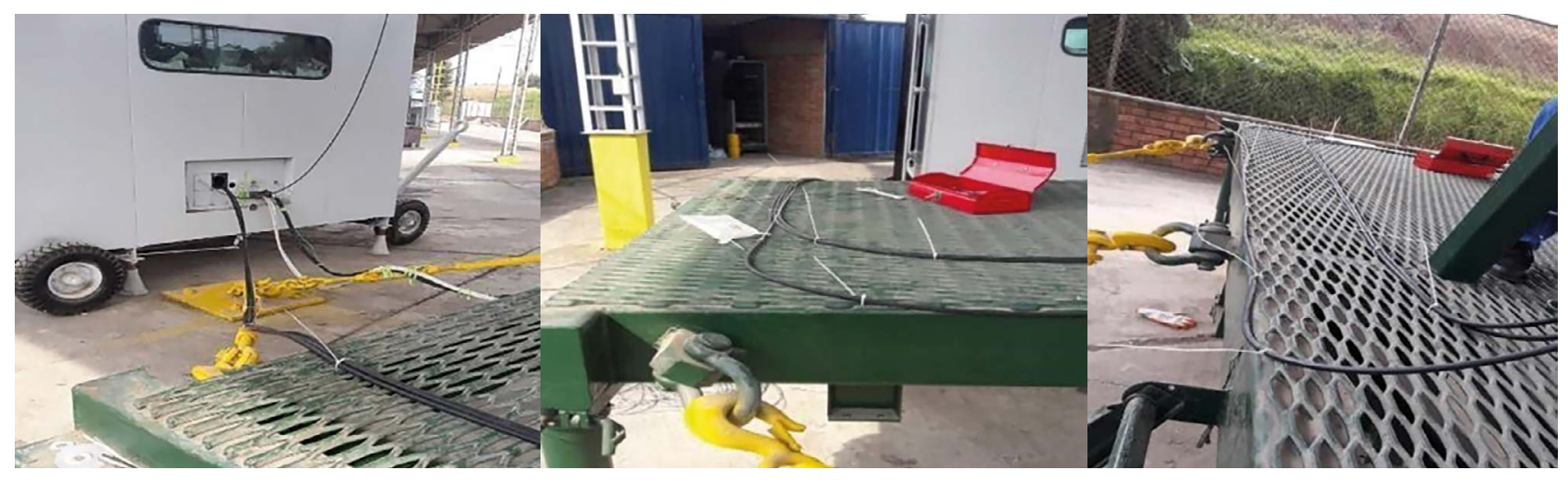

Figura 5. Ruta del cable de control mecánico al banco de prueba Fuente: elaboración propia.

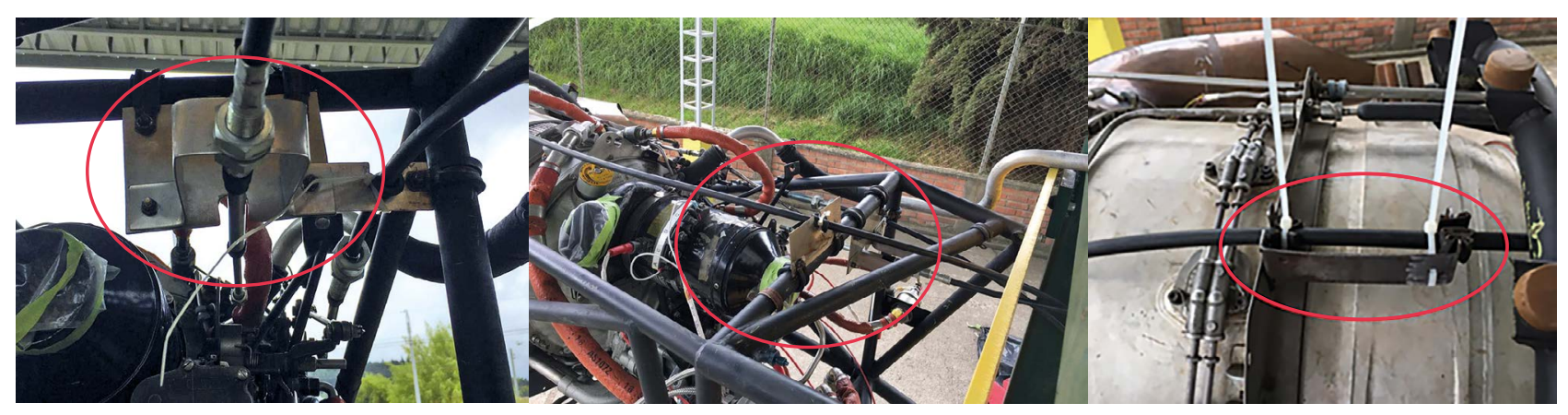

Figura 6. Pasamuros de fijación en el motor Fuente: elaboración propia. 
Los autores del documento fabricaron los elementos en CAMAN, lugar donde se encuentra ubicado el banco de prueba del motor, reutilizando algunas láminas que estaban disponibles en el lugar.

\section{Elaboración de elementos de fijación para el cable de control mecánico en el motor y las palancas}

En la figura 6 se observan los pasamuros construidos para la fijación de los cables de control mecánico en el motor, los cuales están conectados al mando de potencia, mando de hélice y mando de combustible.

Por otra parte, se utilizaron trozos de caucho en el lugar donde el cable estaba apoyado, para así evitar el contacto directo con la lámina y no causar desgastes adicionales en el recubrimiento del cable debido a la fricción con los pasamuros.

\section{Componentes del trazado}

Los principales componentes mecánicos utilizados para la implementación de este controlador mecánico y algunas de sus especificaciones se resumen en la tabla 2.

Tabla 2

Componentes del trazado de control mecánico

\begin{tabular}{l|c|c|c}
\multicolumn{1}{c|}{ Componente } & Cantidad & Longitud & Calibre \\
\hline Terminal tipo rótula & 6 & No aplica & No aplica \\
\hline Pasamuros & 7 & No aplica & No aplica \\
\hline Cable de control mecánico & 2 & 12 metros & $3 / 8$ in $(9,53 \mathrm{~mm})$ \\
\hline Cable de control mecánico & 1 & 14 metros & $3 / 8$ in $(9,53 \mathrm{~mm})$ \\
\hline Extensor tipo telescopio & 3 & $10 \mathrm{~cm}$ & No aplica \\
\hline
\end{tabular}

Fuente: elaboración propia.

\section{Palancas mecánicas}

En el desarrollo de la adecuación del banco de pruebas, se implementó un tipo de comandos diferente que tiene mayor precisión al momento de entrar en operación. Se trata de un conjunto de palancas fabricadas en aluminio, con un recorrido angular de 130 grados que cuentan con topes metálicos, lo que significa un mayor control y, además, permite poner a punto el recorrido de los cables de control mecánico. Las palancas y los elementos que la componen son los que se implementan para el control del motor desde la cabina del banco de pruebas. Dichas palancas fueron adquiridas por la FAC y se pueden apreciar en la siguiente figura:
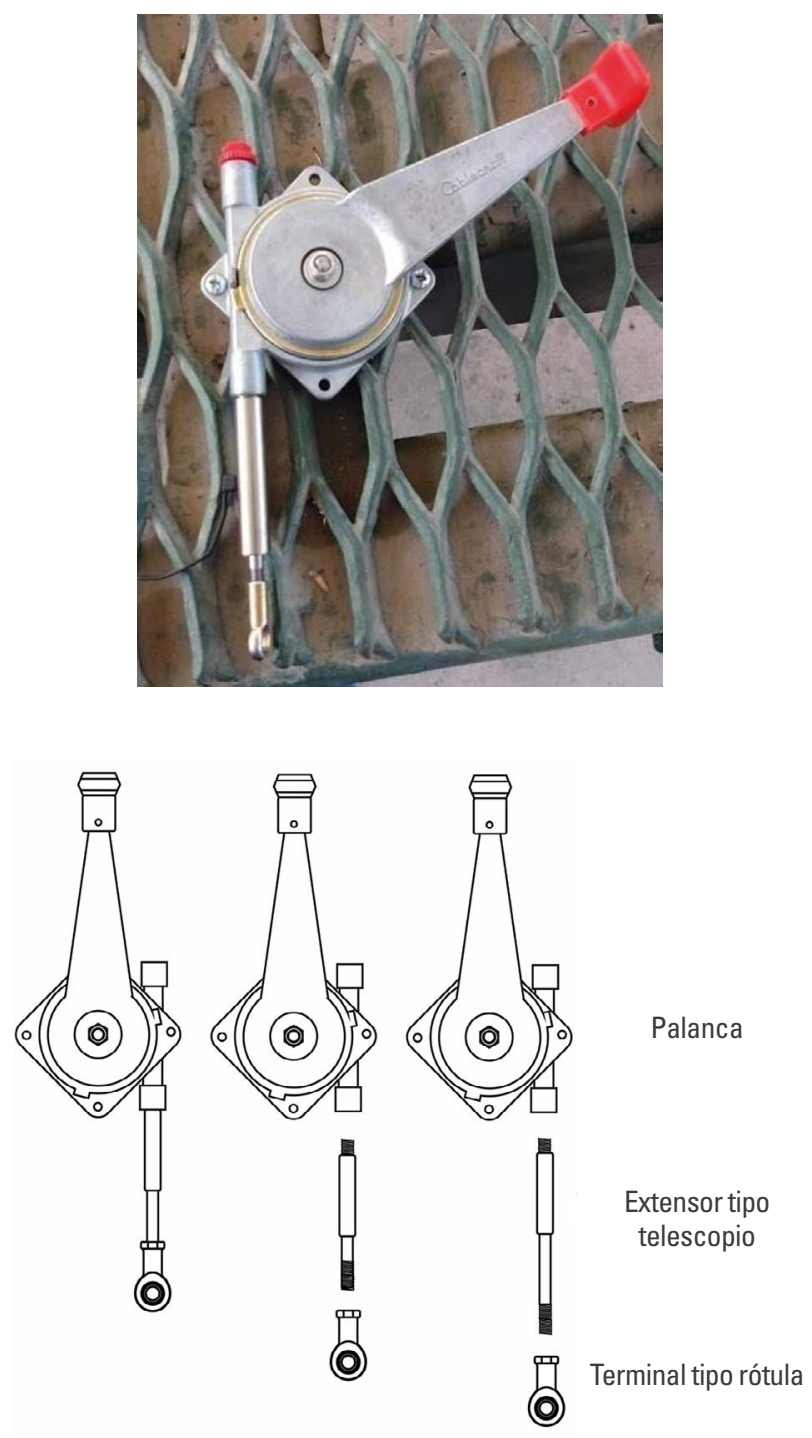

Figura 7. Palanca mecánica y sus componentes Fuente: elaboración propia. 
Este tipo de mandos están instalados en su respectivo compartimiento en el banco de pruebas, el diseño de este banco también cuenta con un acceso que permite revisar la operación de las palancas de comando y los cables. De la misma manera, para facilitar su acople, se instalaron terminales tipo rótula y guías tipo telescopio que entran y salen de la palanca, lo que permite el movimiento de los cables de control $y$, al momento de realizar la acción de control, evita la flexión de la guaya e impide la pérdida de la capacidad de desplazamiento del reglaje. Lo anterior es de vital importancia llegado el momento de la toma de datos, durante las pruebas con los diferentes combustibles y la posterior verificación del comportamiento dinámico del motor.

A continuación, en la figura 8 se observa el tipo de palancas que se utilizan para realizar el control mecánico del motor PT6, y su montaje en la cabina del banco de pruebas.
Las palancas no tienen un criterio de diseño definido, ya que se desconoce la totalidad de sus materiales de fabricación. La lámina empleada tampoco cuenta con especificaciones técnicas, pues es reutilizada al igual que los demás elementos de fijación de los cables a las palancas, esto se refleja en una disminución del costo de implementación, pero puede generar incertidumbres sobre el comportamiento del controlador. Las palancas están ubicadas en la cabina del banco de pruebas para controlar el motor a distancia, con el fin de brindar las condiciones de seguridad necesarias a las personas que realicen experimentos con él.

Para asegurar los cables de control mecánico a estas palancas, se fabricaron elementos de sujeción para la fijación del cable, y así evitar inconvenientes e imprevistos al momento de llevar a cabo las pruebas. Dichos elementos de sujeción se pueden apreciar en la figura 9.
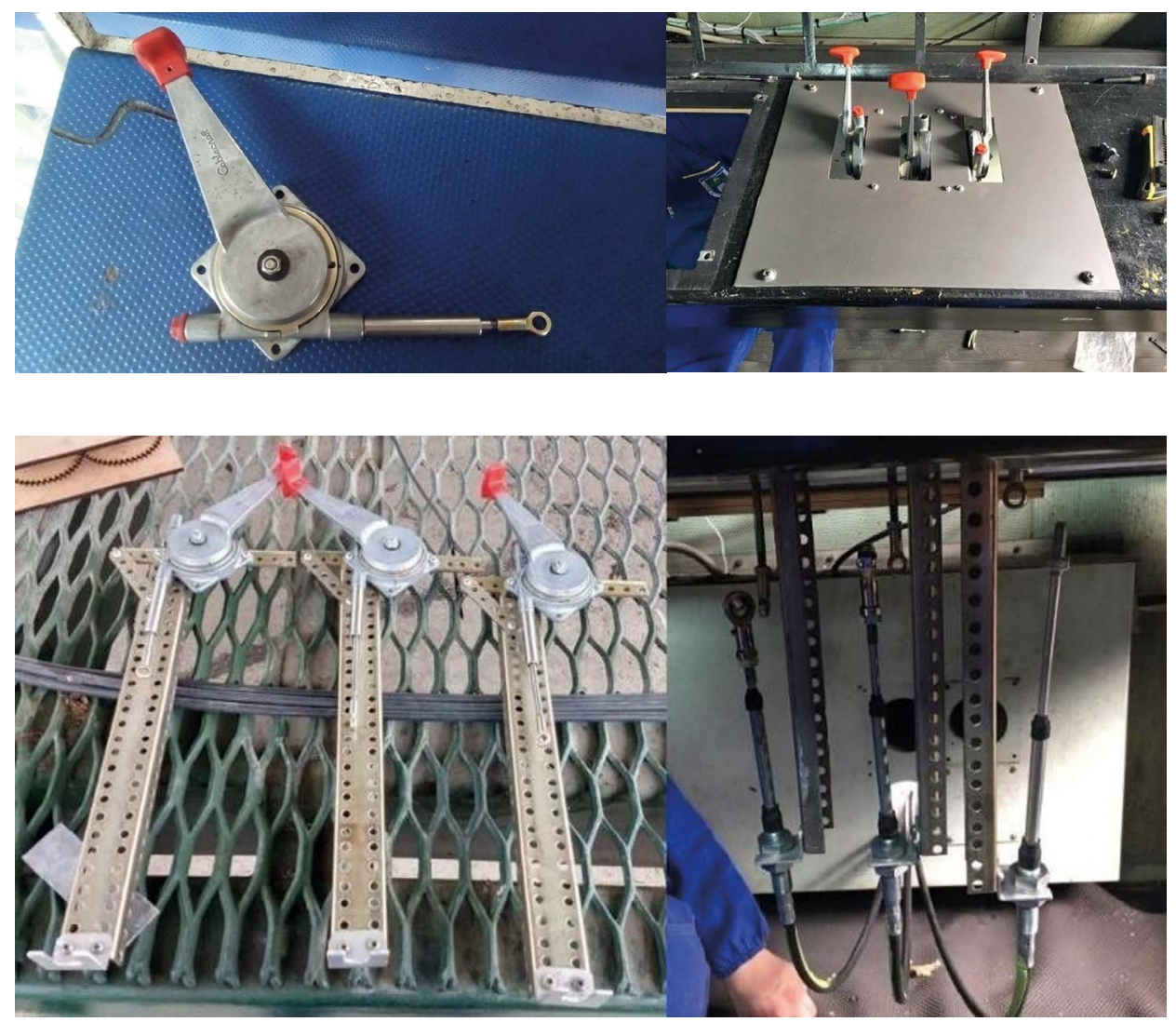

Figura 8. Palanca mecánica Fuente: elaboración propia.

Figura 9. Fijación de los cables de control mecánico a las palancas de control

Fuente: elaboración propia. 
Así, damos paso a la sección de los resultados experimentales que se obtuvieron durante una sesión de encendido y prueba del motor.

\section{Resultados experimentales}

En esta sección se presentan los resultados obtenidos en las pruebas de encendido del motor con las palancas reutilizadas y, a su vez, se observa su comportamiento junto con los cables de control mecánico al momento de accionar las diferentes válvulas de potencia, hélice y combustible.

Figura 10. Esquema de control de potencia Fuente: elaboración propia.
Potencia mín. Reversible

\section{Pruebas de control a los comandos de vuelo}

\section{Palanca de potencia}

En el siguiente esquema se ve cómo es el funcionamiento del cable de control y las palancas que accionan el mando de potencia del motor. Al momento en que el operador del banco de pruebas decida hacer una acción sobre la palanca, el movimiento se verá reflejado en el gobernador de potencia gracias a la conexión del cable de control entre el comando, su correspondiente palanca y la operación del motor.

\section{PALANCA DE POTENCIA \\ PALANCA DE POTENCIA}

cia máx.

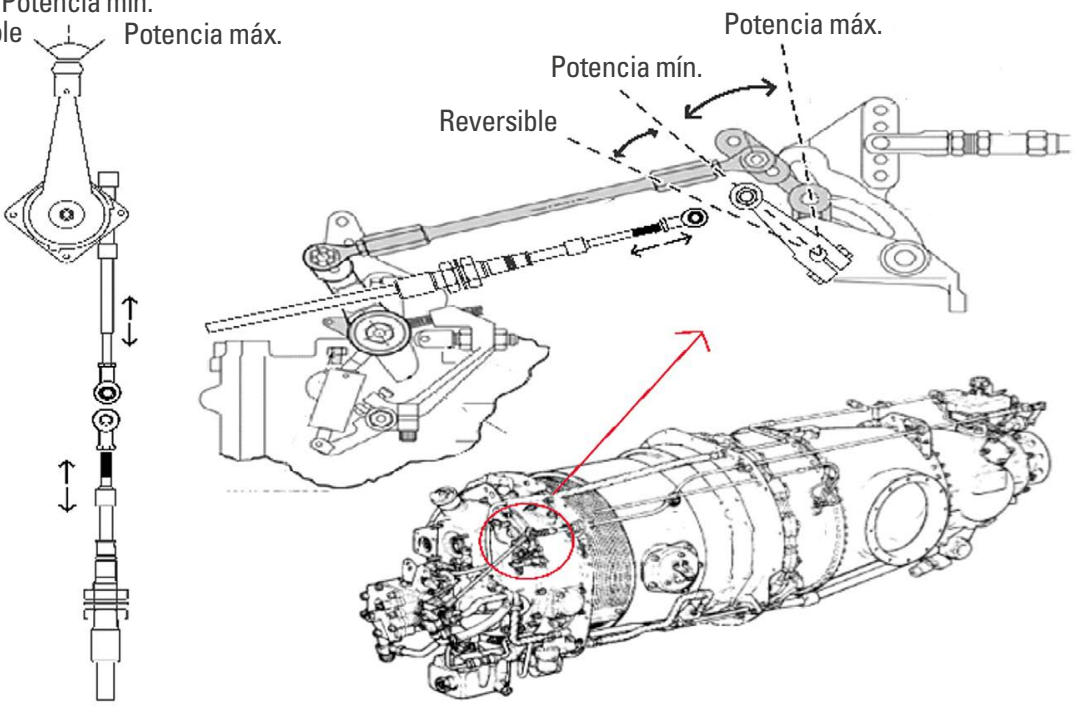

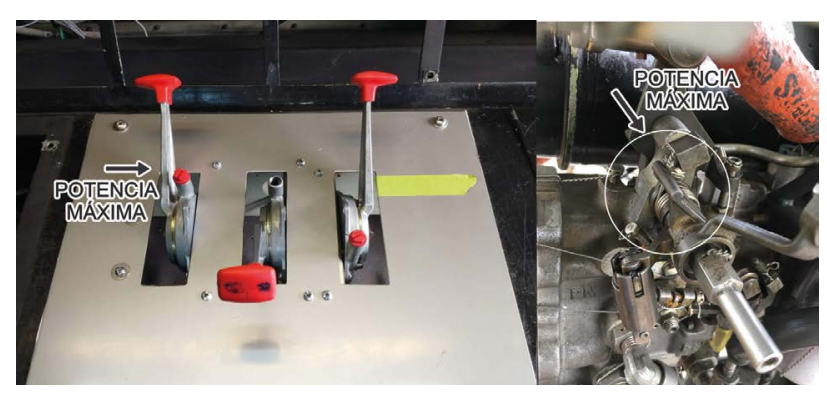

Figura 11. Palancas de potencia y potencia máxima Fuente: elaboración propia.

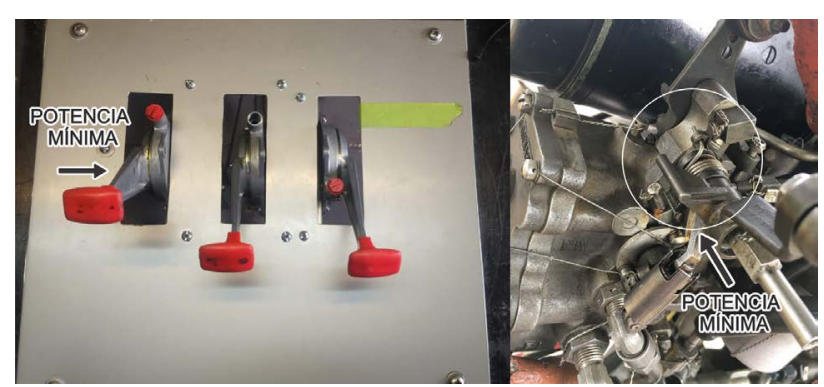

Figura 12. Palancas de potencia y potencia mínima

Fuente: elaboración propia. 
Dada la acción del operador, el conjunto se activará para entrar en los regímenes de funcionamiento del motor de potencia máxima, potencia mínima y reversible, que se pueden ver en las figuras 11 y 12 .

Se observa en la figura 11 la condición de potencia máxima, en que la palanca del lado izquierdo está en posición superior, y en el mando del motor la palanca llega al tope de regulación de máxima potencia del motor.

En la figura 12, la palanca de potencia se encuentra en posición de mínimas junto con el mando del motor, esta posición también es llamada ralentí.

\section{Palanca de paso de hélice}

En el siguiente esquema se ve cómo es el funcionamiento del cable de control y la palanca que acciona el mando de la hélice. Al momento en que el operador del banco de pruebas decida hacer una acción de control, se verá reflejada en el gobernador de hélice y en la operación del motor.

Ahora vemos en las figuras 14 y 15 que el mecanismo es capaz de llegar a los topes, al realizar los cambios en el ángulo de la hélice requeridos al momento de operar el motor.

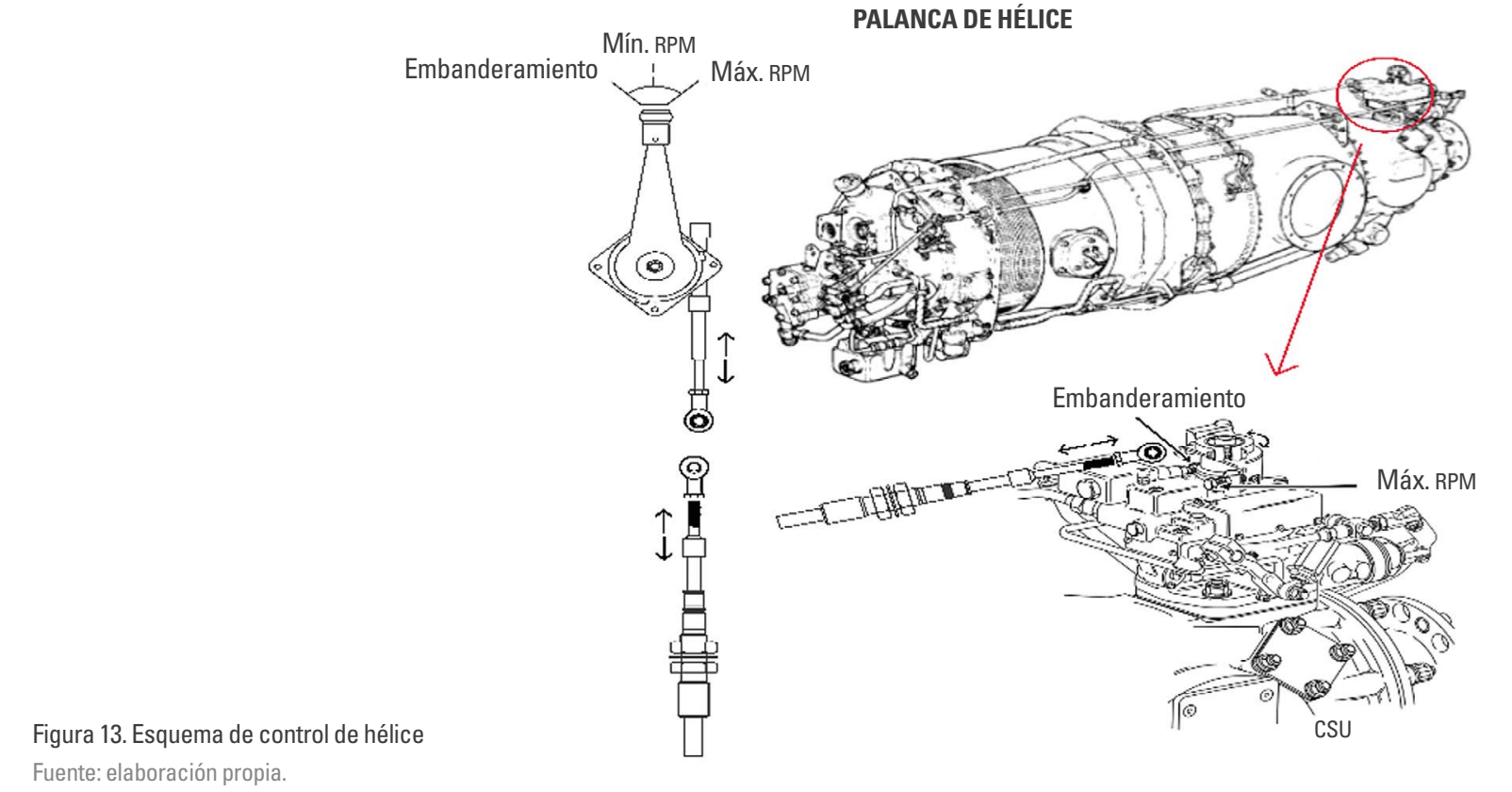

PALANCA DE HÉLICE

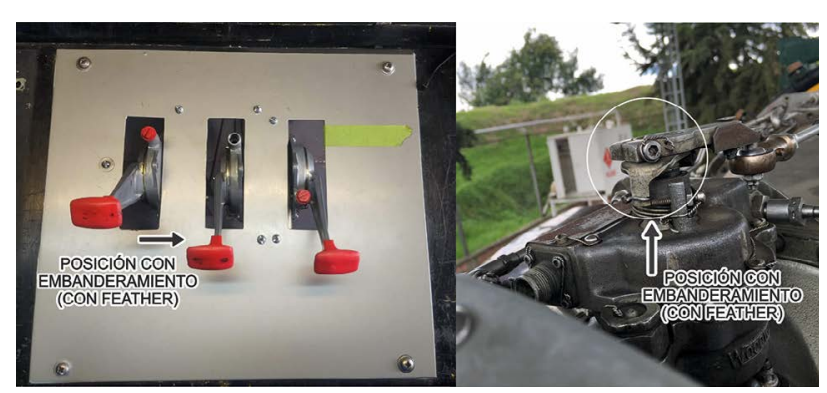

Figura 14. Posición con embanderamiento Fuente: elaboración propia.

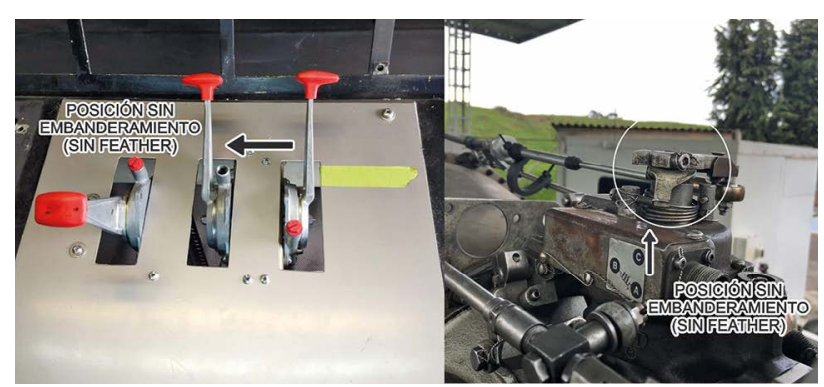

Figura 15. Posición sin embanderamiento

Fuente: elaboración propia. 
Se puede ver en la figura 14 que la segunda palanca es la encargada de variar el paso de la hélice, en esta ocasión, la palanca está en la posición de embanderamiento o feather, condición en la que el motor es capaz de entregar un máximo torque y un mínimo empuje gracias a la posición que toma la hélice.

En la figura 15 se muestra que dicha palanca ya no se encuentra en la posición inicial de embanderamiento, ahora está en una posición en la que la hélice cambia su paso, es decir, sin feather, en la cual el motor es capaz de entregar un mínimo torque y un mayor empuje.

\section{Palanca de flujo de combustible}

En el esquema de la figura 16 se muestra cómo será el funcionamiento del cable de control y su correspondiente palanca, al momento de accionar el mando de combustible del motor. Cuando el operador del banco de pruebas decida hacer una acción con la palanca, se verá reflejado en el gobernador de combustible que operará según sus regímenes y en el control del motor, gracias a la conexión del cable de control entre ellos.

\section{PALANCA DE COMBUSTIBLE}

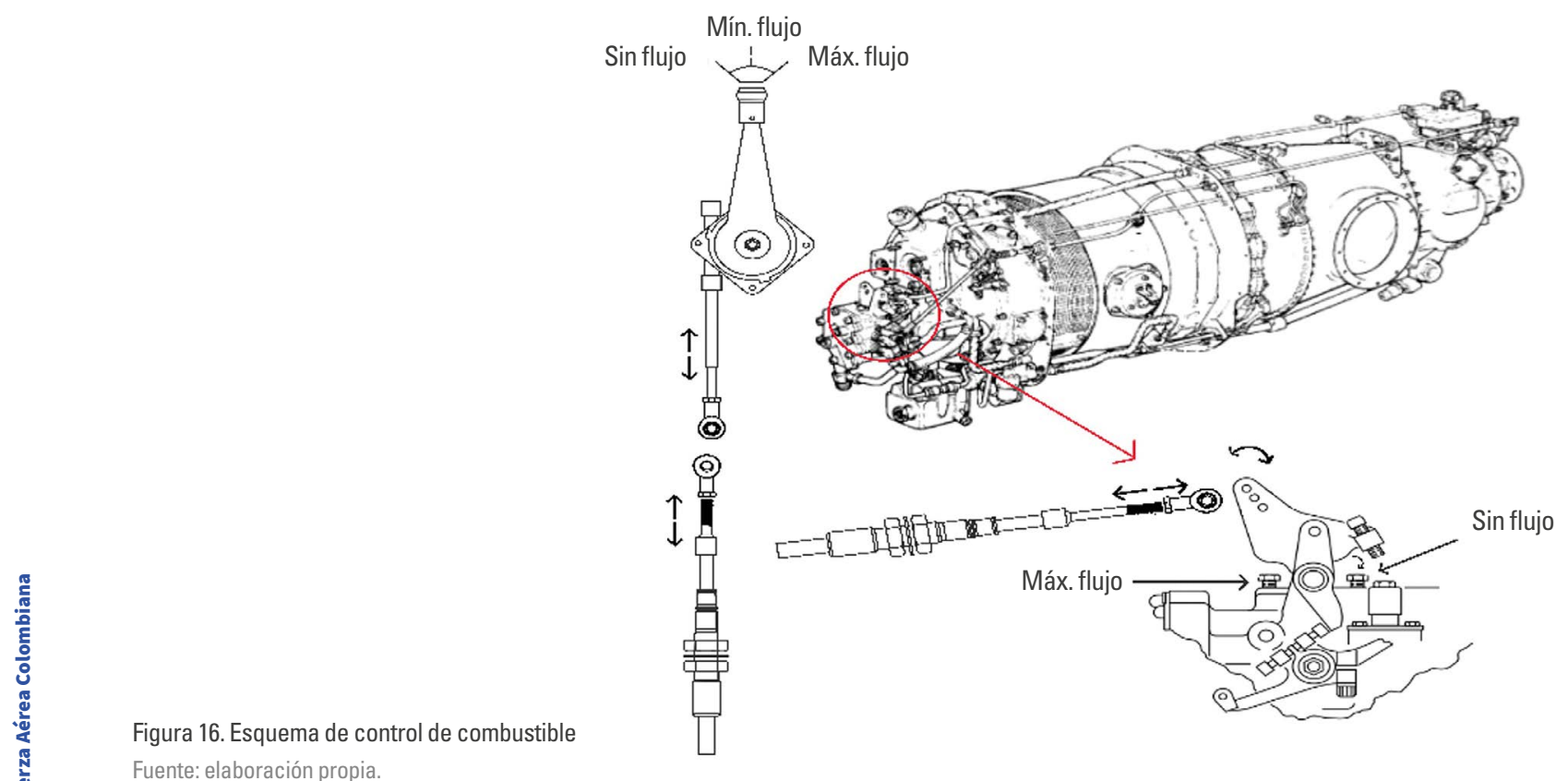

Fuente: elaboración propia.

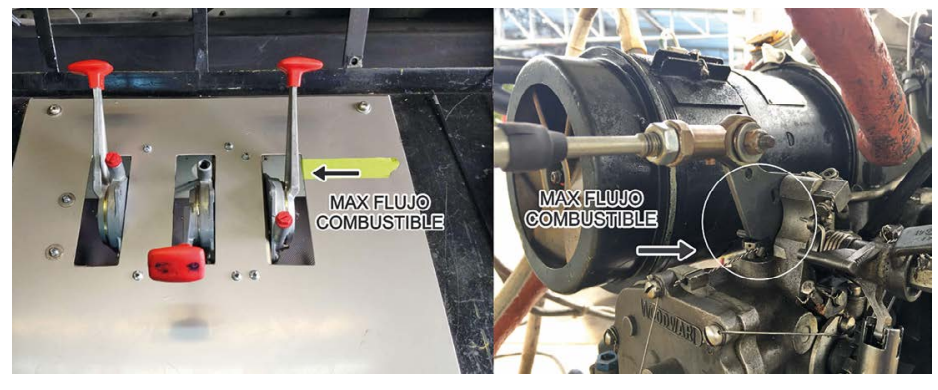

Figura 17. Máximo flujo de combustible Fuente: elaboración propia.

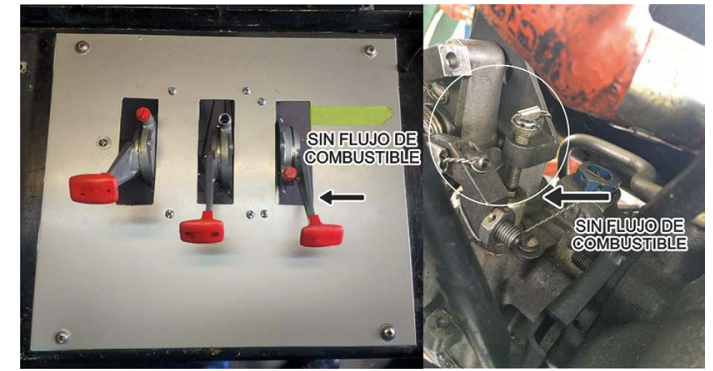

Figura 18. Sin flujo de combustible

Fuente: elaboración propia. 
Ahora bien, respecto al funcionamiento de la palanca que controla el flujo de combustible entrante al motor, se observa que esta, en conjunto con el cable de control mecánico, acciona a la perfección el mando de combustible y le permite llegar a los topes de máximo y cierre de flujo, como se ve en las figuras 17 y 18.

En esta ocasión, se observa la palanca número tres, encargada de controlar el flujo de combustible, que permite o frena el paso de combustible al motor. En la figura 17 la palanca está en la posición de paso de combustible, a la vez que el mando de accionamiento del motor se encuentra en el tope de máximo flujo de combustible.

La figura 18 muestra que la palanca está en la posición que no permite el flujo de combustible al motor, $y$ en el mando de accionamiento se observa que el cable de control mecánico está en el tope inferior, es decir, en el que no es posible suministrar combustible al motor.

En último lugar, lo que resta es ejecutar pruebas con JET A-1 y biodiesel para verificar experimentalmente los reglajes de los tres comandos de vuelo y el desempeño del motor PT6-61A, que se presenta en la siguiente sección.

\section{Reglajes teóricos y prácticos del cable de control mecánico}

Se presenta el resumen de los reglajes, tanto teóricos como prácticos en la tabla 3. En ella es posible evidenciar que con el $100 \%$ del avance en las palancas se obtiene un movimiento angular menor al esperado con los cálculos teóricos. Sin embargo, el motor consigue llegar a la potencia máxima. El combustible y el ángulo de hélice logran alcanzar sus topes respectivos.

Como se puede apreciar, existe una inconsistencia en el recorrido angular de las palancas entre los reglajes teóricos y prácticos. Esto se debe a que no fue posible calibrar con suficiente precisión el recorrido de los comandos del motor, así como también el juego en los acoples de las rótulas influyó en el error de esta medida.
Tabla 3

Reglajes teóricos y prácticos del cable de control mecánico

Reglajes teóricos de las palancas de comando

\begin{tabular}{l|c|c}
\hline Comando & Avance de la palanca (\%) & Grados de recorrido \\
\hline Potencia & 100 & $130^{\circ}$ \\
\hline Combustible & 100 & $130^{\circ}$ \\
\hline Hélice & 100 & $130^{\circ}$
\end{tabular}

Reglajes prácticos de las palancas de comando

\begin{tabular}{l|c|c} 
Comando & Avance de la palanca (\%) & Grados de recorrido \\
\hline Potencia & 100 & $125^{\circ}$ \\
\hline Combustible & 100 & $130^{\circ}$ \\
\hline Hélice & 100 & $130^{\circ}$
\end{tabular}

Fuente: elaboración propia.

\section{Pruebas de operación en tierra del controlador mecánico}

Después de la implementación del control mecánico a través de los cables y palancas vistas, se realizaron pruebas en el motor con combustible tradicional ET A-1, algunas mezclas entre este y un biocombustible con porcentajes de 5,15\% y $25 \%$ de biodiesel. En las pruebas se destacó que el motor alcanzó un $96 \%$ de potencia durante su operación en la sección generadora de gases (NG), con una velocidad máxima de 1990 rpm en la hélice (NP) y la generación de un torque de $1600 \mathrm{lb} / \mathrm{ft}$. Fue posible además, alcanzar una velocidad en reversible de 1735 rpm en NP con un promedio de $73 \%$ en NG, y un torque de $134 \mathrm{lb} / \mathrm{ft}$. Por último, la velocidad en mínimas fue de $1300 \mathrm{rpm}$ en NP, con un promedio de $63 \%$ en NG y con un torque de $37,37 \mathrm{lb} / \mathrm{ft}$. Estos datos son un estimado de la totalidad de la corrida experimental realizada, el lector interesado en profundizar en estos resultados, puede consultarlos en la investigación de Sánchez (2020).

\section{Discusión}

Como se observó en la tabla 3 sobre reglajes teóricos y prácticos de los cables de control mecánico y sus 
palancas, al momento de realizar la manipulación en una de las tres palancas del banco de prueba se presentó un pequeño porcentaje de error. Esto se debe a que algunos de los artículos empleados para realizar el acople de los elementos originalmente presentan algún tipo de holgura, como es el caso de las rótulas dispuestas en las terminales del cable de control mecánico Push-Pull. Sin embargo, es posible y seguro realizar las pruebas con este porcentaje mínimo de error, ya que, a pesar de estar presente en la palanca de potencia (con un valor de $5^{\circ}$ de desplazamiento angular), el motor se puede llevar a su operación mínima y máxima sin ningún tipo de esfuerzo añadido.

Los resultados de desempeño del controlador mecánico presentados durante las pruebas experimentales en cuanto a velocidad, torque y porcentaje de potencia en el eje, son los esperados en un tipo de prueba como la descrita, lo que se muestra en el éxito del controlador propuesto y en el promedio de valores expuesto.

\section{Observaciones y recomendaciones}

Se describen a continuación las observaciones y recomendaciones pertinentes para la ejecución de una segunda fase del proyecto, en lo referente a la realización repetitiva de ensayos en tierra, y se enuncian las recomendaciones de seguridad a tener en cuenta para las pruebas que se han de realizar al motor desde el banco de pruebas, para así no tener inconvenientes al momento de la operación del motor en tierra.

\section{Observaciones}

Es destacable que la gran mayoría de los materiales empleados para la elaboración de pasamuros, anclajes de palancas, entre otros, son materiales reutilizados, lo que representa una disminución significativa del presupuesto asignado para la compra de estos elementos. Por otra parte, dada la naturaleza y el tiempo de entrega del proyecto, no se realizó un estudio de fuerzas en los cables, siendo imprescindible su realización con el fin de analizar el comportamiento de los cables de control mecánico y verificar que no estén siendo sometidos a esfuerzos o fricciones que puedan ocasionar daños futuros en el controlador o en el motor.

\section{Recomendaciones}

Las recomendaciones que se consideran necesarias para el buen funcionamiento de este controlador son, en primera instancia, verificar periódicamente el ajuste de los elementos de fijación presentes en el trazado del cable de control mecánico, para obtener un rango de operación eficiente. Segundo, revisar el comportamiento de los cables de control mecánico al momento de estar en operación, para así evidenciar su correcto funcionamiento y evitar movimientos no deseados. Por último, es necesario llevar un seguimiento de los pasamuros, ya que el caucho empleado para evitar el desplazamiento de los cables de control puede sufrir algún tipo de desgaste y esto, a su vez, generar daños en los cables.

\section{Conclusión}

Dada la implementación lograda en el banco de prueba fue posible posicionar el recorrido de los cables de control mecánico en el banco, y así obtener una respuesta con un porcentaje de error mínimo, a saber, igual al $4 \%$ en el comando de potencia y del $0,1 \%$ en los dos comandos restantes, dando la confiabilidad necesaria requerida para la realización de las pruebas a futuro. Así mismo, se obtuvo el rendimiento máximo y mínimo de cada comando, lo cual facilita la toma de datos en cada prueba con diferentes porcentajes de mezcla. Hay que mencionar que se logra una repetitividad favorable de los ensayos llevados a cabo con este control mecánico, sin la presencia de porcentajes de error drásticos en la conversión del movimiento angular a lineal. Para concluir, gracias a los elementos de fijación construidos, se logran asegurar los cables de control mecánico en la bancada del motor y en el interior de la cabina del banco de pruebas para operación en tierra. 


\section{Referencias bibliográficas}

Badger, M., Julien, A., LeBlanc, A., Moustapha, S., Prabhu, A., \& Smailys, A. (1994, 1 de abril). The PT6 Engine: 30 Years of Gas Turbine Technology Evolution. ASME. J. Eng. Gas Turbines Power, 116, 322-330. https://doi.org/10.1115/ 1.2906823

Bayona-Roa, C., Solís-Chaves, J. S., Bonilla, J., RodríguezMeléndez, A., \& Castellanos, D. (2019). Computational Simulation of PT6A Gas Turbine Engine Operating with Different Blends of Biodiesel-A Transient-Response Analysis. Energies, 12(22). https://doi.org/10.3390/en1 2224258

BioD. (2015). Biodiesel. http://www.biodsa.com.co/index.php/ premium-gold/?lang=es

Cables y Controles. (2020). Cables de tire y empuje. http:// www.cablesycontroles.com/catalogo-de-productos/ cables/cables-de-tire-y-empuje/

Delgado, P. (2018, 2 de septiembre). En Colombia hay industria aeronáutica. El Espectador. https://www.elespectador.com/economia/en-colombia-hay-industria-aero nautica-articulo-809827

Ferreira, G., Jurado, E., \& Ayala, J. (2008). Diseño de un banco de pruebas para motores PT6T-3 [Tesis de pregrado]. Universidad de San Buenaventura. http://biblioteca.usbbog.edu.co:8080/Biblioteca/BDigital/43285.pdf

Fuerza Aérea Colombiana. (s.f.). Cheyenne III \& III A PA-42 720. https://www.fac.mil.co/media-gallery/detail/8011/5350

Guayacol. (2013). Guayas y cables de control. https://guayacol. com/index.php/productos/guayas-y-cables-de-control. html

Guimarães, Diogo Maria (2015). Implementation of an Engine Condition Trend Monitoring (ECTM) Program in a Part $M$ Organization: Pratt \& Whitney PT6A-67D Engine [Tesis de maestría]. Universidade Da Beira Interior. https:// pdfs.semanticscholar.org/d219/7ce753781d2753a6a32 680dc5f8aa3339e72.pdf?_ga=2.85481881.1057691772. 1585602801-1237244739.1585602801

Mayorga Betancourt, M. A., Cadavid Estrada, J. G., Bonilla Páez, J. A., López Santamaría, C. A., \& López Gómez, M. (2019). Use of Biofuels in the Aeronautical Industry: Case of the Colombian Air Force. TECCIENCIA, 14(26), 53-63. http://dx. doi.org/10.18180/tecciencia.2019.26.7

Meisel, A. (2014, marzo). Volando sobre la ruta de los vapores: los comienzos de SCADTA, 1919-1930. Revista Credencial. http://www.revistacredencial.com/credencial/historia/ temas/volando-sobre-la-ruta-de-los-vapores-los-comienzos-de-scadta-1919-1930

Méndez, A. (2011). Elaboración de un manual interactivo de entrenamiento en el sistema de arranque del motor PT6 [Tesis de tecnología]. Instituto Tecnológico Superior Aeronáutico. https://repositorio.espe.edu.ec/bitstream/21000/8585/1/T-ESPE-ITSA-000156.pdf

Pratt \& Whitney Canada. (2019). PT6A More than a Motor. https://www.pwc.ca/en/products-and-services/products/ general-aviation-engines/pt6a

Pratt \& Whitney Canada. (2007). PT6A-60 Series Training Manual. https://mikeklochcfi.files.wordpress.com/2018/08/ training-pt6a-60-series.pdf

Pratt \& Whitney Canada. (2006). Cost-Effective PT6A Overhaul Solutions. https://n9.cl/egta

Pratt \& Whitney Canada. (s.f.). Key New Order Wisdow Your PT6A Turboprop. https://www.caijets.com/pdf/KnowYourPT6A.pdf

Sánchez, A. P. (2020, mayo). Desarrollo de pruebas y análisis experimental de un motor PT6A-61A de la Fuerza Aérea Colombiana a condiciones de Bogotá, realizadas en banco de ensayos utilizando JET A-1 y biodiesel [Tesis de pregrado no publicada]. Universidad ECCI.

Semana. (2018, 1 de octubre). La industria aeronáutica de Colombia cada vez vuela más alto. Semana. https://n9.cl/i840 